\title{
Spontaneous intercostal artery bleeding in a patient with alcohol-induced liver cirrhosis
}

\author{
Mandeep Singh Rahi ${ }^{1}$, Prachi Pednekar ${ }^{1}$, Gaurav Parmar ${ }^{1}$, Lauren Keibel $^{1}$, Kulothungan \\ Gunasekaran $^{1}$, Kwesi Amoah ${ }^{1}$, and Christopher Winterbottom ${ }^{1}$ \\ ${ }^{1}$ Bridgeport Hospital
}

January 11, 2021

\begin{abstract}
An adult male with alcoholic cirrhosis presented with acute blood loss anemia, hypotension and a painful bruise over his body's left side. He denied any trauma or falls. Computed tomography of the chest showed left chest wall hematoma. Emergent angiography with successful embolization of the left intercostal artery was performed.
\end{abstract}

\section{Spontaneous intercostal artery bleeding in a patient with alcohol-induced liver cirrhosis}

Mandeep Singh Rahi, MD ${ }^{1}$; Prachi Pednekar, $\mathrm{MD}^{2}$; Gaurav Parmar, $\mathrm{MD}^{3}$; Lauren Keibel, DO ${ }^{1}$; Kulothungan Gunasekaran, $\mathrm{MD}^{1}$; Kwesi Amoah, $\mathrm{MD}^{1}$; Christopher Winterbottom, $\mathrm{MD}^{1}$

${ }^{1}$ Division of Pulmonary Diseases and Critical Care Medicine, Yale-New Haven Health Bridgeport Hospital, Bridgeport, CT, USA

${ }^{2}$ Department of Internal Medicine, Yale-New Haven Health Bridgeport Hospital, Bridgeport, CT, USA

${ }^{3}$ Department of Radiology, Yale-New Haven Health Bridgeport Hospital, Bridgeport, CT, USA

Correspondence to:

Mandeep Singh Rahi, MD

Division of Pulmonary Diseases and Critical Care Medicine

Yale-New Haven Health Bridgeport Hospital

267 Grant Street

Bridgeport, CT 06610, USA

Phone: 571-314-1212

Fax: 203-330-7498

Email: rahi.mandeepsingh@yahoo.com

A 44-year-old male with alcoholic cirrhosis presented with one day of fatigue. He noticed a painful bruise over his body's left side but denied any trauma or falls. On arrival, he was hypotensive with a hemoglobin of $2.7 \mathrm{~g} / \mathrm{dL}$, platelet count of $46,000 / \mathrm{mm}^{3}$, and INR of 2.04. Computed tomography of the chest and abdomen revealed a large intramuscular hematoma within the left lateral chest wall extending to the upper abdomen measuring $12 \mathrm{~cm} \mathrm{x} 5 \mathrm{~cm}$ and several foci of high attenuation suggestive of acute hemorrhage (Figures 1 and 2 ). In addition to resuscitation, emergent angiography was performed. Active extravasation from the left 
ninth intercostal artery was noted. Successful embolization with 500 to 700 -micron embospheres followed by gel foam slurry was performed (Figures 3 and 4). Spontaneous intercostal artery bleeding is rare in cirrhosis, with few cases reported so far. ${ }^{1}$ Risk factors, in addition to thrombocytopenia and clotting factor deficiency in cirrhotic patients, are alcohol use, trauma, hypertension, and anticoagulation. ${ }^{2}$ Management of closed space spontaneous bleeding in cirrhotic patients is challenging. In mild cases, medical management with judicious use of blood products should suffice. Severe cases are managed with transcatheter arterial embolization and, ultimately, liver transplantation.

\section{REFERENCES}

1. Seon CS, Jo Y, Lee J, et al. Spontaneous Arterial Bleeding in Patients with Alcoholic Liver Cirrhosis: a Series of Three Cases.Korean J Med . 10 2014;87(4):449-454. doi:10.3904/kjm.2014.87.4.449

2. Gunasekaran K, Rajasurya V, Devasahayam J, et al. A Review of the Incidence Diagnosis and Treatment of Spontaneous Hemorrhage in Patients Treated with Direct Oral Anticoagulants. J Clin Med . Sep 15 2020;9(9)doi:10.3390/jcm9092984

\section{FIGURE LEGENDS}

Figure 1: A computed tomography of the chest and abdomen in the coronal view showing a large intramuscular hematoma within the left lateral chest wall and extending to the upper abdomen, denoted by a star.

Figure 2: A computed tomography of the chest and abdomen in the axial view showing foci of high attenuation in the intramuscular hematoma involving the left lateral chest wall suggestive of acute bleeding, denoted by arrows.

Figure 3: Angiographic image of the left intercostal arteries showing active extravasation from the ninth intercostal artery, denoted by arrows.

Figure 4: Angiographic image of the left intercostal arteries showing no extravasation after successful embolization of the night intercostal artery. 

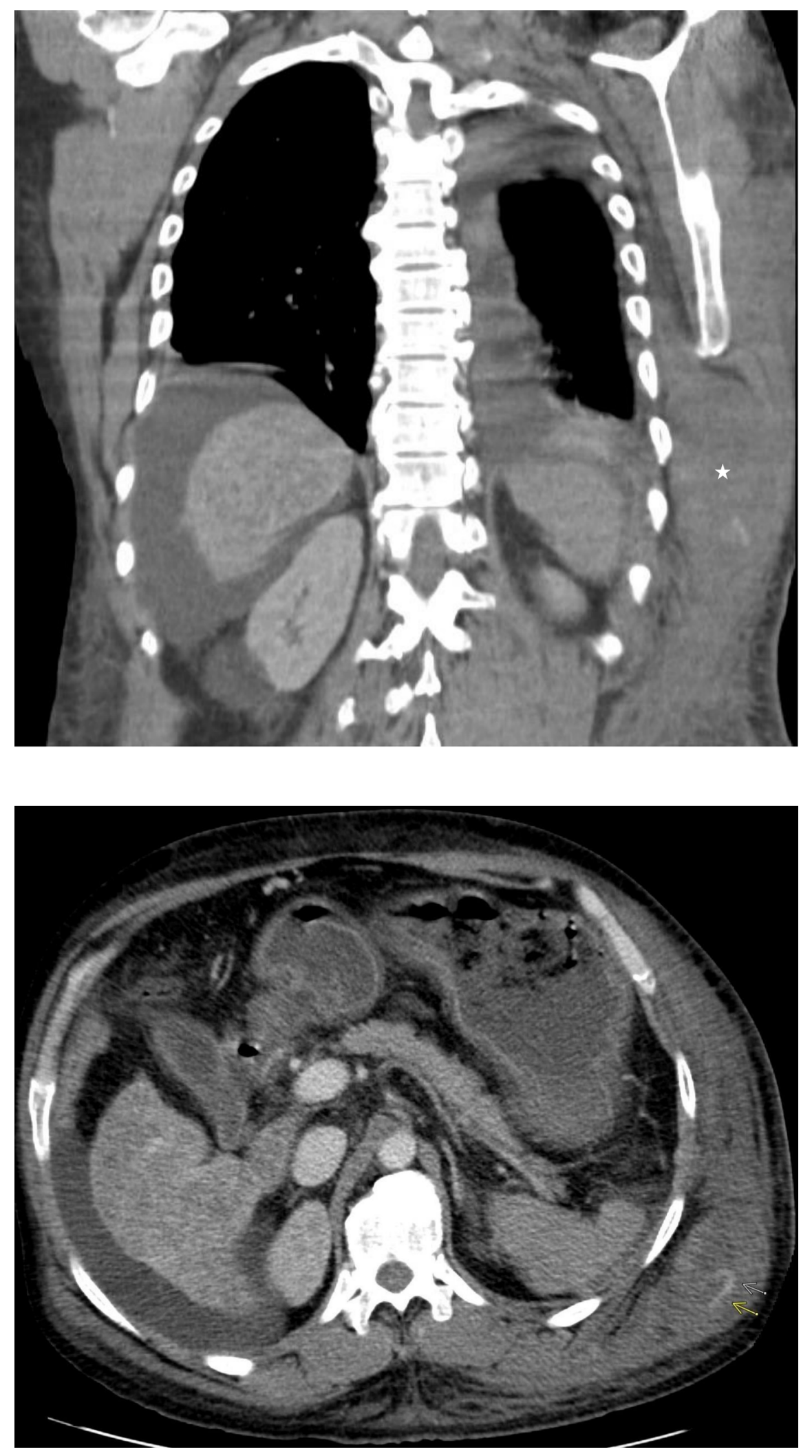


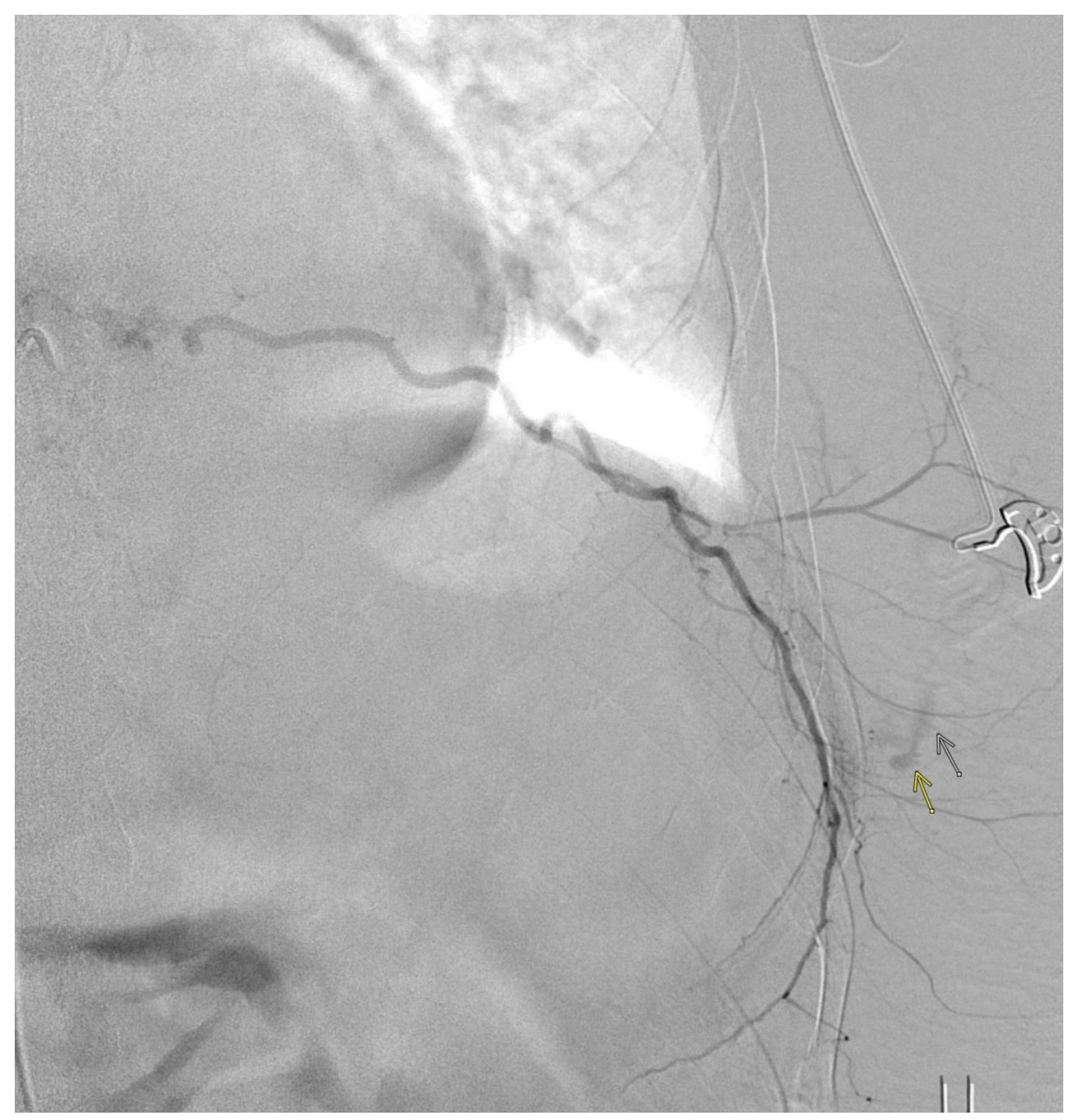




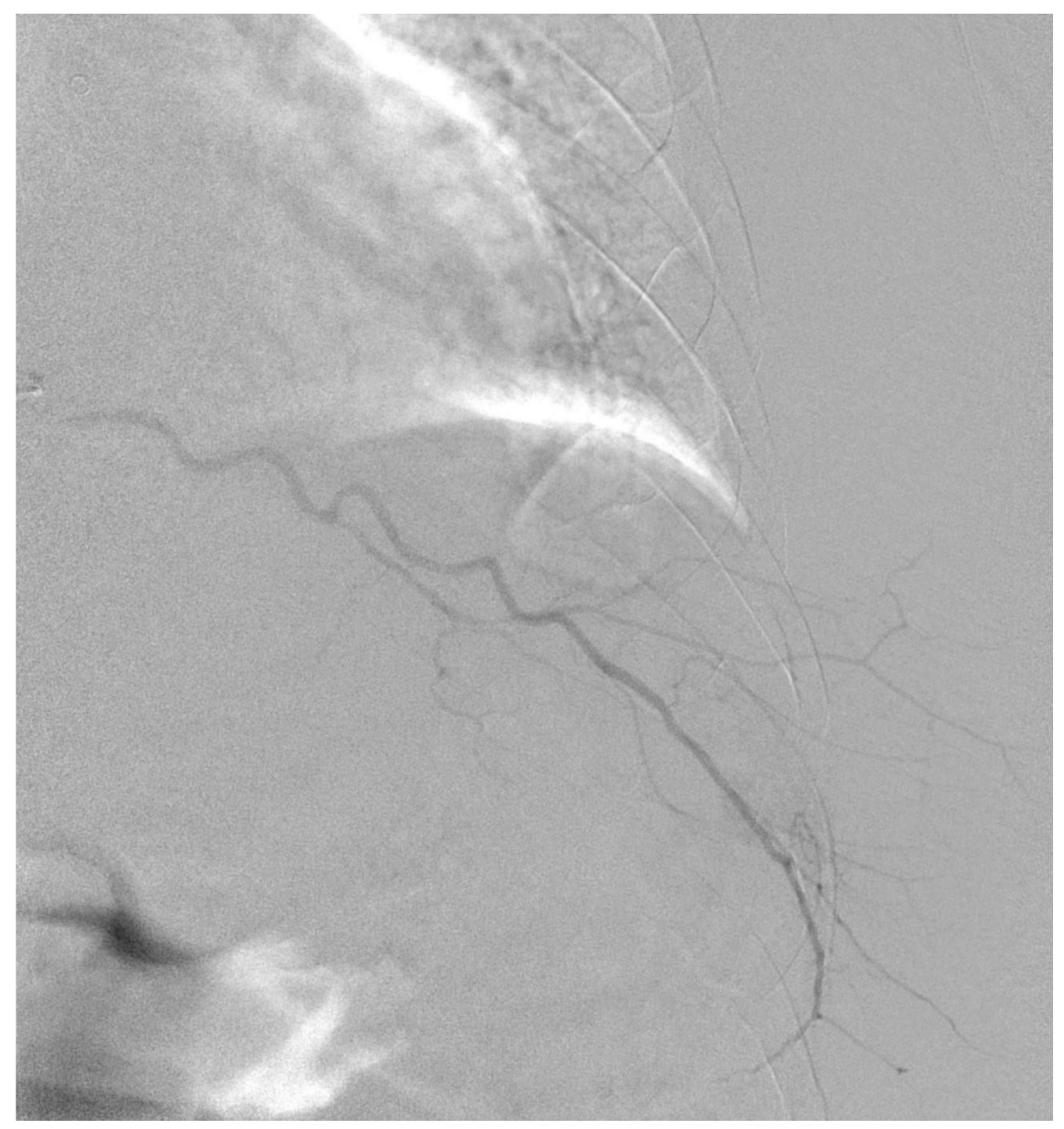

